\title{
NHC-Palladium (II) Mononuclear and Binuclear Complexes Containing Phenylene-bridged Bis(thione) Ligands: Synthesis, Characterization and Catalytic Activities
}

Wei-Guo Jia, ${ }^{\text {a,c }}$ Li-Li Gao ${ }^{\mathrm{a}}$, Zhi-Bao Wang ${ }^{\mathrm{a}}$, Jing-Jing Wanga ${ }^{\mathrm{a}}$, En-Hong Sheng ${ }^{\mathrm{a}}$, and Ying-Feng Han*b

a: The Key Laboratory of Functional Molecular Solids, Ministry of Education, Anhui Laboratory of Molecular-Based Materials (State Key Laboratory Cultivation Base), College of Chemistry and Materials Science, Anhui Normal University, Wuhu, 241002, China

b: Key Laboratory of Synthetic and Natural Functional Molecular Chemistry of Ministry of Education, College of Chemistry and Materials Science, Northwest University, Xi'an 710127, China.

c: State Key Laboratory of Structural Chemistry, Fujian Institute of Research on the Structure of Matter, Chinese Academy of Science, Fuzhou 350002, China.

Corresponding Author

*E-mail: wgjiasy@mail.ahnu.edu.cn (W.G. Jia)

*E-mail:yfhan@nwu.edu.cn (Y. F. Han).

\section{Contents}

1. Table S1 Crystallographic data and structure refinement parameters for Pd complexes.

2. Table S2 Selected bond lengths $(\AA)$ and bond angels $\left(^{\circ}\right)$ for Pd complexes.

3. Copies of ${ }^{1} \mathrm{H},{ }^{13} \mathrm{C}$ NMR Spectra of palladium complexes 1a-3b.

4. ESI-MS spectra of palladium complexes 1a-3b 
Table S1 Crystallographic data and structure refinement parameters for Pd complexes a

\begin{tabular}{|c|c|c|c|}
\hline & $1 b$ & $2 a$ & $3 \mathbf{b}$ \\
\hline Empirical formula & $\mathrm{C}_{43} \mathrm{H}_{54} \mathrm{I}_{2} \mathrm{~N}_{12} \mathrm{OPd}_{2} \mathrm{~S}_{4}$ & $\mathrm{C}_{47} \mathrm{H}_{46} \mathrm{I}_{2} \mathrm{~N}_{12} \mathrm{O}_{2} \mathrm{Pd}_{2} \mathrm{~S}_{4}$ & $\mathrm{C}_{26} \mathrm{H}_{32} \mathrm{~F}_{6} \mathrm{~N}_{6} \mathrm{PPdS} \mathrm{S}_{2}$ \\
\hline Formula weight & 1349.82 & 1405.80 & 744.06 \\
\hline Crystal syst., Space group & Monoclinic; $\mathrm{P} 2{ }_{1} / \mathrm{c}$ & Monoclinic; $\mathrm{C} 2 / \mathrm{c}$ & Monoclinic; $\mathrm{P} 2{ }_{1} / \mathrm{n}$ \\
\hline $\mathrm{a}(\AA)$ & $25.4628(4)$ & 25.9641(9) & $15.952(3)$ \\
\hline $\mathrm{b}(\AA)$ & $7.35067(15)$ & $7.0268(3)$ & $8.193(2)$ \\
\hline c $(\AA)$ & $27.2943(5)$ & $31.0957(12)$ & $24.073(5)$ \\
\hline$\beta\left(^{\circ}\right)$ & $96.6153(16)$ & $100.063(3)$ & $93.19(3)$ \\
\hline Volume $\left(\AA^{3}\right), \mathrm{Z}$ & $5074.63(17) ; 4$ & $5586.0(4) ; 4$ & 3141.3(12), 4 \\
\hline $\mathrm{D}_{\mathrm{c}}\left(\mathrm{mg} / \mathrm{m}^{3}\right)$ & 1.767 & 1.672 & 1.573 \\
\hline$\mu(\mathrm{Mo}-\mathrm{K} \alpha)\left(\mathrm{mm}^{-1}\right)$ & 17.180 & 1.946 & 0.838 \\
\hline $\mathrm{F}(000)$ & 2664 & 2760 & 1508 \\
\hline $2 \theta$ range $\left(^{\circ}\right)$ & $7.034-134.142$ & $6.374-50.018$ & $5.802-50.054$ \\
\hline Reflections/unique; [R(int)] & $18967 / 9016 ;[R($ int $)=0.0495]$ & $14038 / 4802 ;[\mathrm{R}(\mathrm{int})=0.0293]$ & $78667 / 5540 ;[\mathrm{R}(\mathrm{int})=0.1723]$ \\
\hline Data/restraints/parameters & $9016 / 774 / 587$ & $4802 / 7 / 325$ & $5540 / 47 / 383$ \\
\hline Goodness-of-fit on $\mathrm{F}^{2}$ & 1.067 & 1.062 & 1.128 \\
\hline$R_{1}, w R_{2}[I>2 s(I)]^{\mathrm{a}}$ & $\mathrm{R}_{1}=0.0690, \mathrm{wR}_{2}=0.1766$ & $\mathrm{R}_{1}=0.0345, \mathrm{wR}_{2}=0.0787$ & $\mathrm{R}_{1}=0.0944 ; \mathrm{wR}_{2}=0.1634$ \\
\hline$R_{1}, w R_{2}$ (all data) & $\mathrm{R}_{1}=0.0734, \mathrm{wR}_{2}=0.1804$ & $\mathrm{R}_{1}=0.0401, \mathrm{wR}_{2}=0.0820$ & $\mathrm{R}_{1}=0.1640 ; \mathrm{wR}_{2}=0.1909$ \\
\hline
\end{tabular}

a: $\mathrm{R}_{1}=\Sigma|| F_{\mathrm{o}}|-| F_{\mathrm{c}}|| \Sigma\left|F_{\mathrm{o}}\right| ; \mathrm{wR}_{2}=\left[\Sigma w\left(\left|F_{\mathrm{o}}^{2}\right|-\left|F_{\mathrm{c}}^{2}\right|\right)^{2} / \Sigma w\left|F_{\mathrm{o}}^{2}\right|^{2}\right]^{1 / 2}$ 
Table S2 Selected bond lengths $(\AA)$ and bond angels $\left({ }^{\circ}\right)$ for Pd complexes

\begin{tabular}{|c|c|c|c|}
\hline \multicolumn{4}{|l|}{$1 b$} \\
\hline $\operatorname{Pd}(1)-C(1)$ & $2.035(9)$ & $\operatorname{Pd}(2)-C(22)$ & $2.052(9)$ \\
\hline $\operatorname{Pd}(1)-C(17)$ & $2.063(8)$ & $\operatorname{Pd}(2)-C(38)$ & $2.081(9)$ \\
\hline $\operatorname{Pd}(1)-S(1)$ & $2.300(2)$ & $\operatorname{Pd}(2)-S(3)$ & $2.324(2)$ \\
\hline $\operatorname{Pd}(1)-S(2)$ & $2.332(2)$ & $\mathrm{Pd}(2)-\mathrm{S}(4)$ & $2.316(2)$ \\
\hline$C(7)-S(1)$ & $1.709(9)$ & $C(33)-S(3)$ & $1.721(9)$ \\
\hline $\mathrm{C}(12)-\mathrm{S}(2)$ & $1.708(9)$ & $\mathrm{C}(28)-\mathrm{S}(4)$ & $1.696(9)$ \\
\hline$C(1)-P d(1)-S(1)$ & $90.1(3)$ & $C(1)-P d(1)-C(17)$ & $172.9(4)$ \\
\hline$C(1)-P d(1)-S(2)$ & $88.7(3)$ & $C(17)-P d(1)-S(1)$ & $90.6(2)$ \\
\hline$S(1)-P d(1)-S(2)$ & $178.72(8)$ & $C(17)-P d(1)-S(2)$ & $90.6(2)$ \\
\hline$C(22)-P d(2)-S(3)$ & $89.6(2)$ & $\mathrm{C}(22)-\mathrm{Pd}(2)-\mathrm{C}(38)$ & $178.0(3)$ \\
\hline$C(22)-P d(2)-S(4)$ & $89.6(2)$ & $C(38)-P d(2)-S(3)$ & $89.4(3)$ \\
\hline$S(3)-P d(2)-S(4)$ & $177.49(7)$ & $\mathrm{C}(38)-\mathrm{Pd}(2)-\mathrm{S}(4)$ & $91.5(3)$ \\
\hline \multicolumn{4}{|l|}{$2 a$} \\
\hline $\operatorname{Pd}(1)-C(1)$ & $2.046(4)$ & $\mathrm{Pd}(1)-\mathrm{S}(2)$ & $2.3374(10)$ \\
\hline $\operatorname{Pd}(1)-C(15)$ & $2.065(4)$ & $\mathrm{C}(7)-\mathrm{S}(1)$ & $1.717(4)$ \\
\hline $\operatorname{Pd}(1)-S(1)$ & $2.3145(10)$ & $C(11)-S(2)$ & $1.711(4)$ \\
\hline$C(1)-P d(1)-S(1)$ & $88.64(10)$ & $C(1)-P d(1)-C(15)$ & $178.85(15)$ \\
\hline$C(1)-P d(1)-S(2)$ & $89.71(10)$ & $C(15)-P d(1)-S(1)$ & $90.94(11)$ \\
\hline$S(1)-P d(1)-S(2)$ & $178.20(4)$ & $C(15)-P d(1)-S(2)$ & $90.70(11)$ \\
\hline \multicolumn{4}{|l|}{$3 \mathbf{b}$} \\
\hline $\operatorname{Pd}(1)-C(3)$ & $2.006(9)$ & $\mathrm{Pd}(1)-\mathrm{S}(2)$ & $2.282(3)$ \\
\hline $\operatorname{Pd}(1)-C(21)$ & $2.022(8)$ & $\mathrm{C}(1)-\mathrm{S}(1)$ & $1.690(10)$ \\
\hline $\operatorname{Pd}(1)-S(1)$ & $2.279(3)$ & $C(5)-S(2)$ & $1.650(11)$ \\
\hline $\mathrm{C}(3)-\mathrm{Pd}(1)-\mathrm{S}(1)$ & $90.9(3)$ & $C(3)-P d(1)-C(21)$ & $175.2(4)$ \\
\hline$C(3)-P d(1)-S(2)$ & $88.9(3)$ & $\mathrm{C}(21)-\mathrm{Pd}(1)-\mathrm{S}(1)$ & $91.8(3)$ \\
\hline$S(1)-P d(1)-S(2)$ & $176.72(11)$ & $\mathrm{C}(21)-\mathrm{Pd}(1)-\mathrm{S}(2)$ & $88.6(3)$ \\
\hline
\end{tabular}




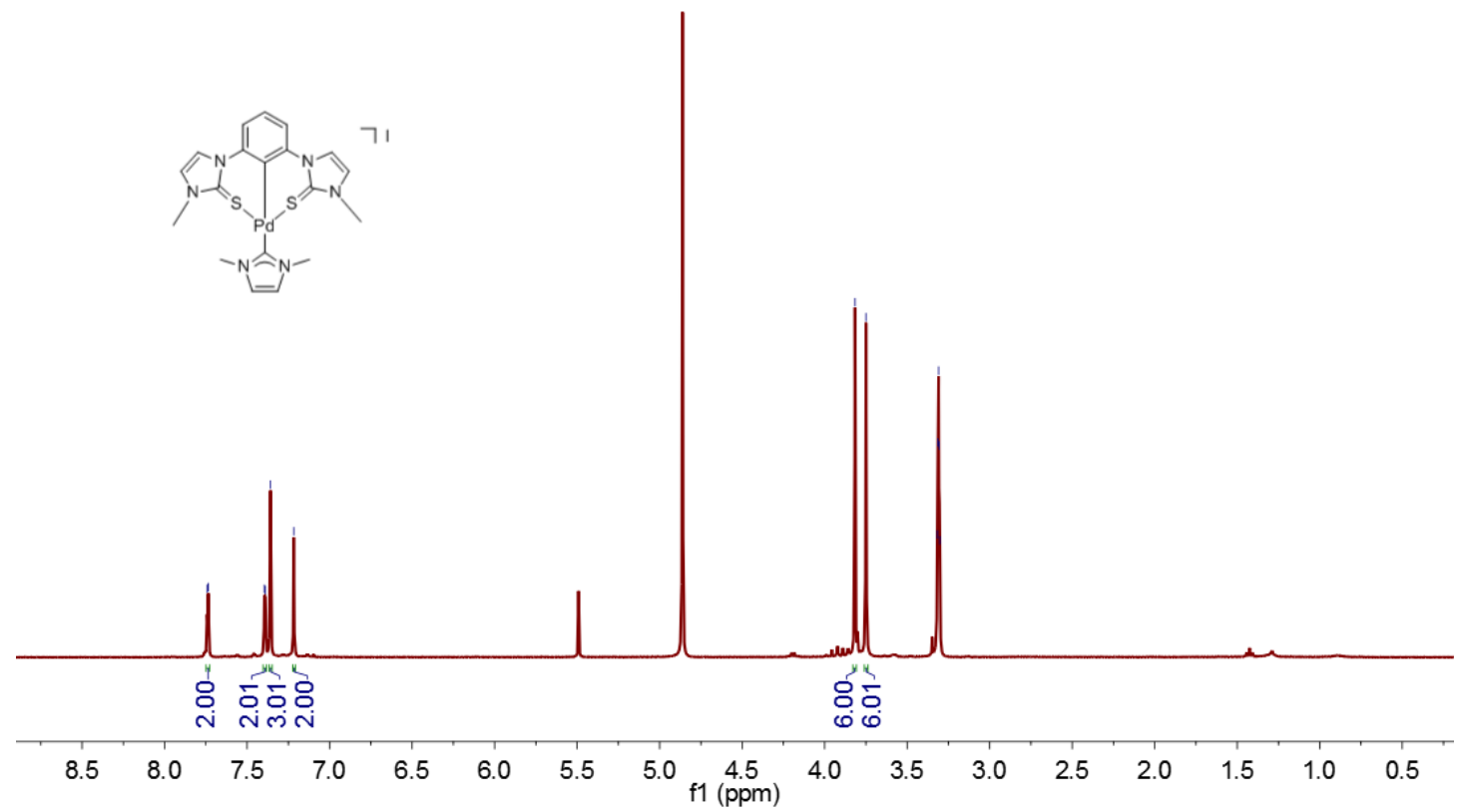

Figure S1. The ${ }^{1} \mathrm{H}$ NMR of 1a $\left(400 \mathrm{MHz}, \mathrm{MeOD}-\mathrm{d}_{4}\right)$

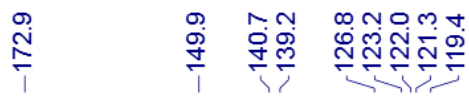

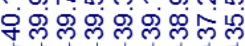

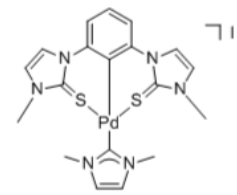

Figure S2. The ${ }^{13} \mathrm{C}$ NMR of $1 \mathrm{a}\left(100 \mathrm{MHz}, \mathrm{DMSO}-\mathrm{d}_{6}\right)$ 


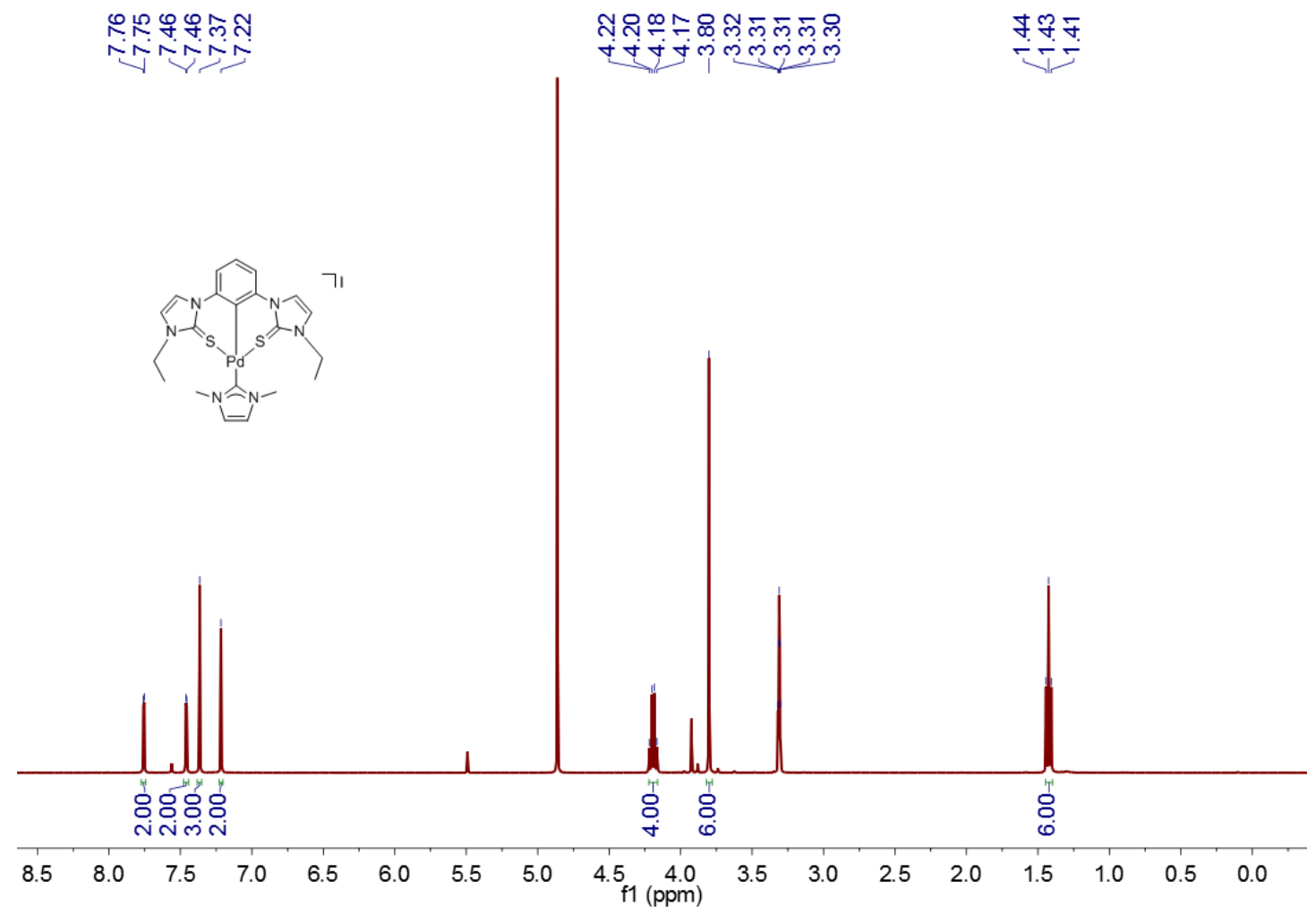

Figure S3.The ${ }^{1} \mathrm{H}$ NMR of $\mathbf{1 b}\left(400 \mathrm{MHz}, \mathrm{MeOD}-\mathrm{d}_{4}\right)$
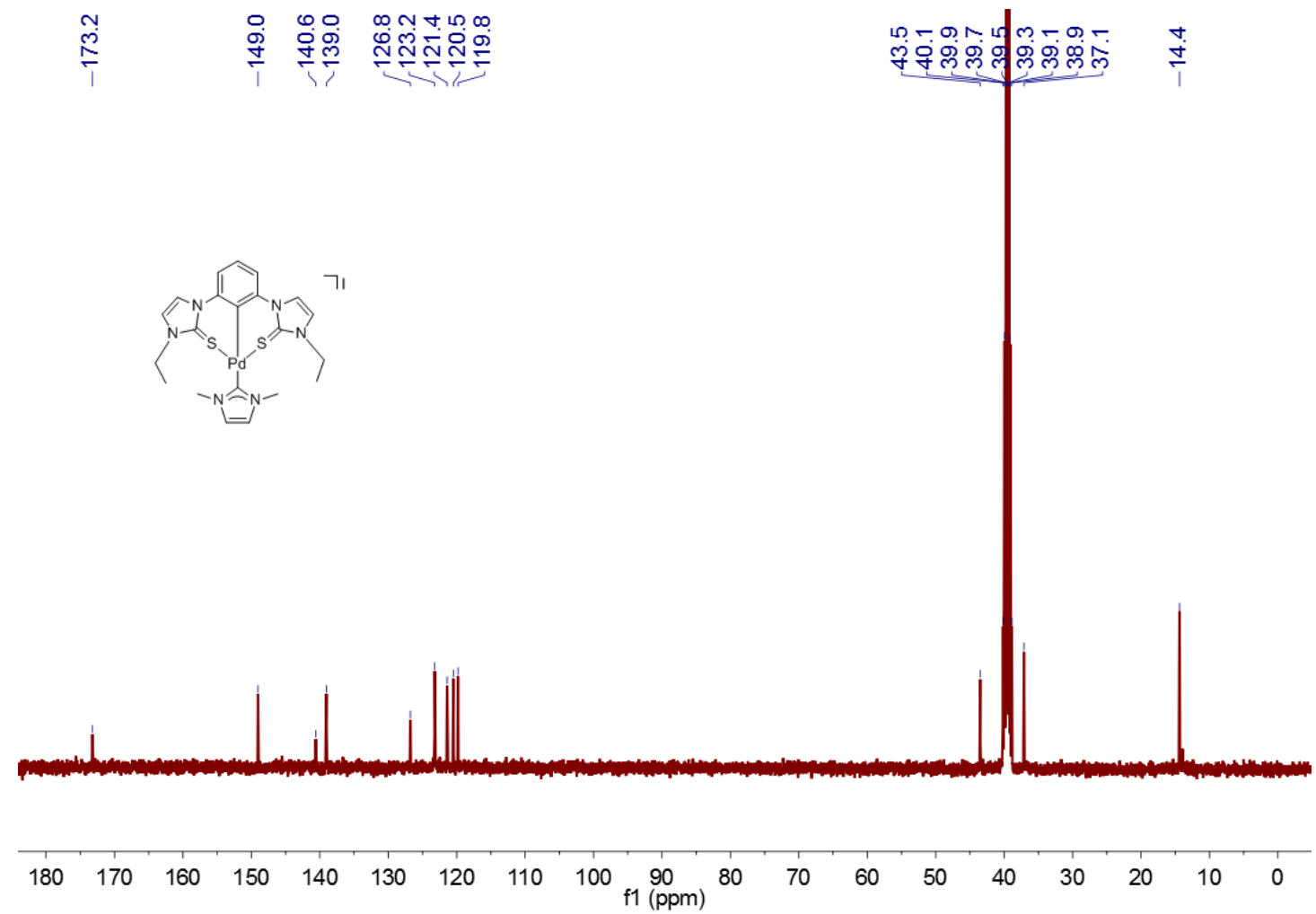

Figure S4. The ${ }^{13} \mathrm{C}$ NMR of $\mathbf{1 b}\left(100 \mathrm{MHz}, \mathrm{DMSO}-\mathrm{d}_{6}\right)$ 


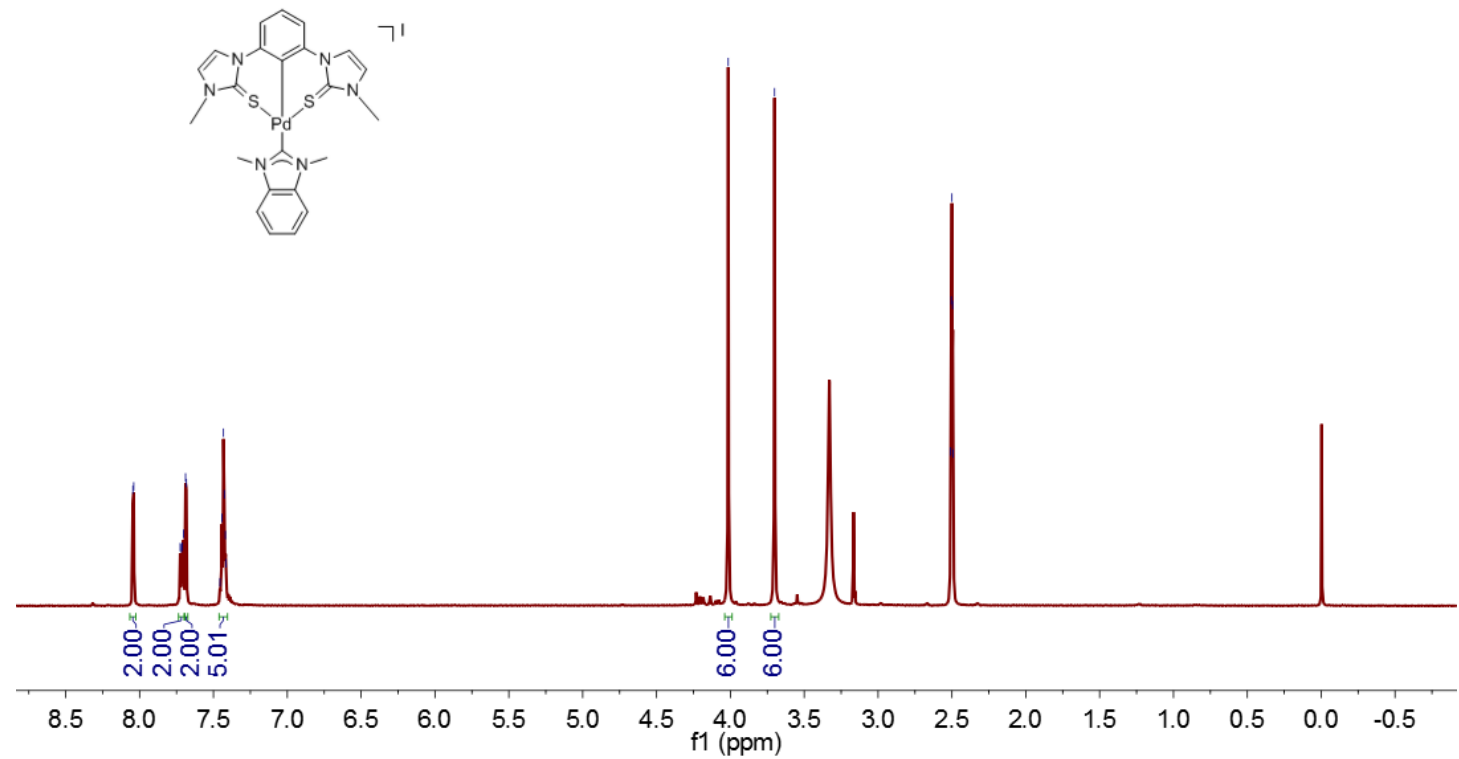

Figure S5. The ${ }^{1} \mathrm{H}$ NMR of 2a $\left(400 \mathrm{MHz}, \mathrm{DMSO}-\mathrm{d}_{6}\right)$

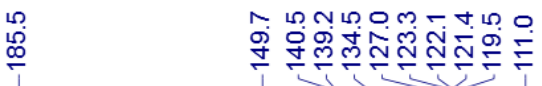

नं नंनं कं

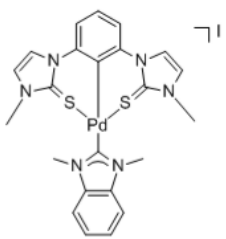

$\begin{array}{lllllllllllllllllllll}200 & 190 & 180 & 170 & 160 & 150 & 140 & 130 & 120 & \begin{array}{r}110 \\ \mathrm{f}(\mathrm{ppm})\end{array} & 90 & 80 & 70 & 60 & 50 & 40 & 30 & 20 & 10 & 0\end{array}$

Figure S6. The ${ }^{13} \mathrm{C}$ NMR of 2a $\left(100 \mathrm{MHz}, \mathrm{DMSO}-\mathrm{d}_{6}\right)$ 


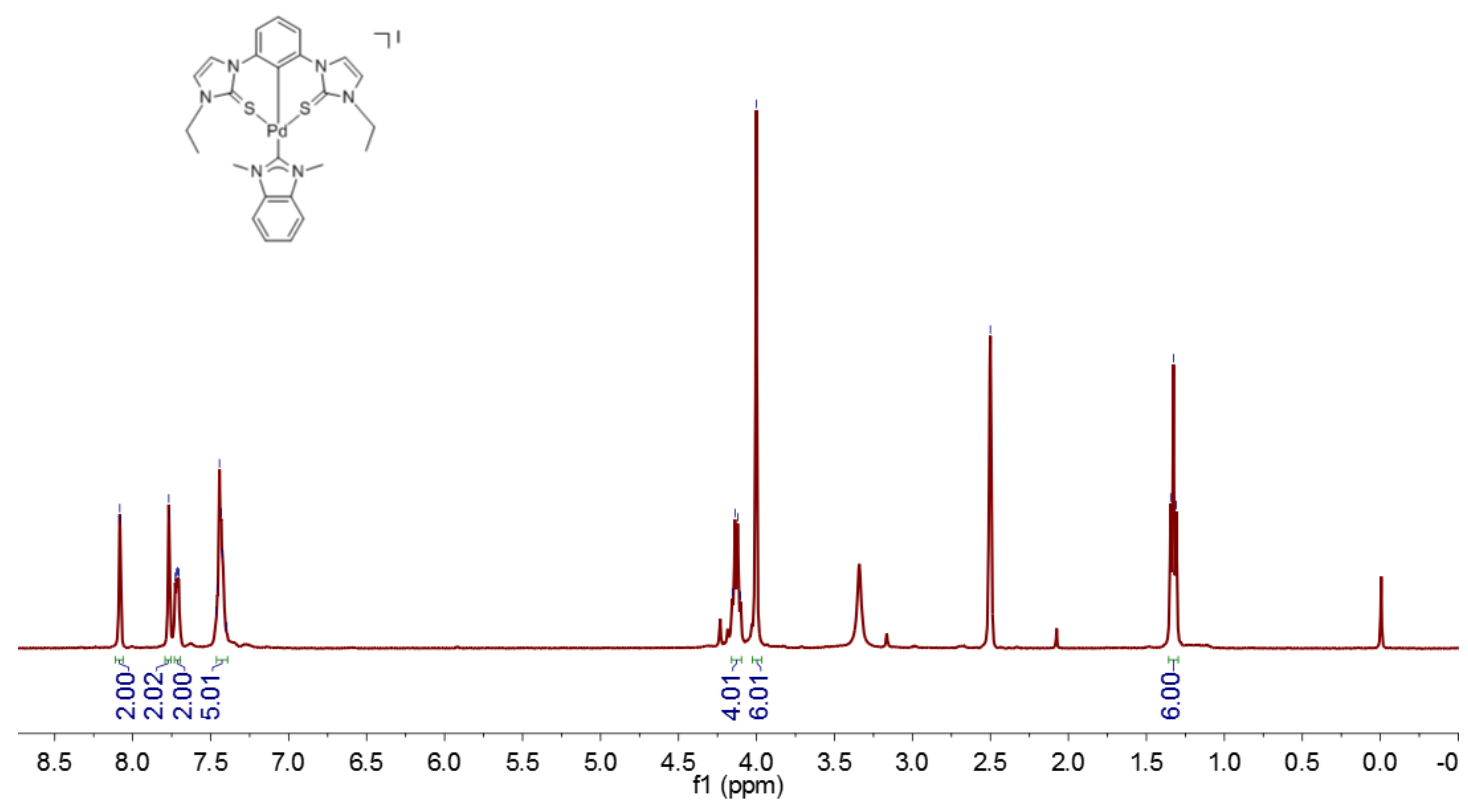

Figure S7. The ${ }^{1} \mathrm{H}$ NMR of $\mathbf{2 b}\left(400 \mathrm{MHz}, \mathrm{DMSO}_{-} \mathrm{d}_{6}\right)$

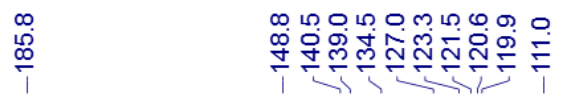

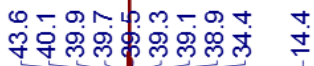
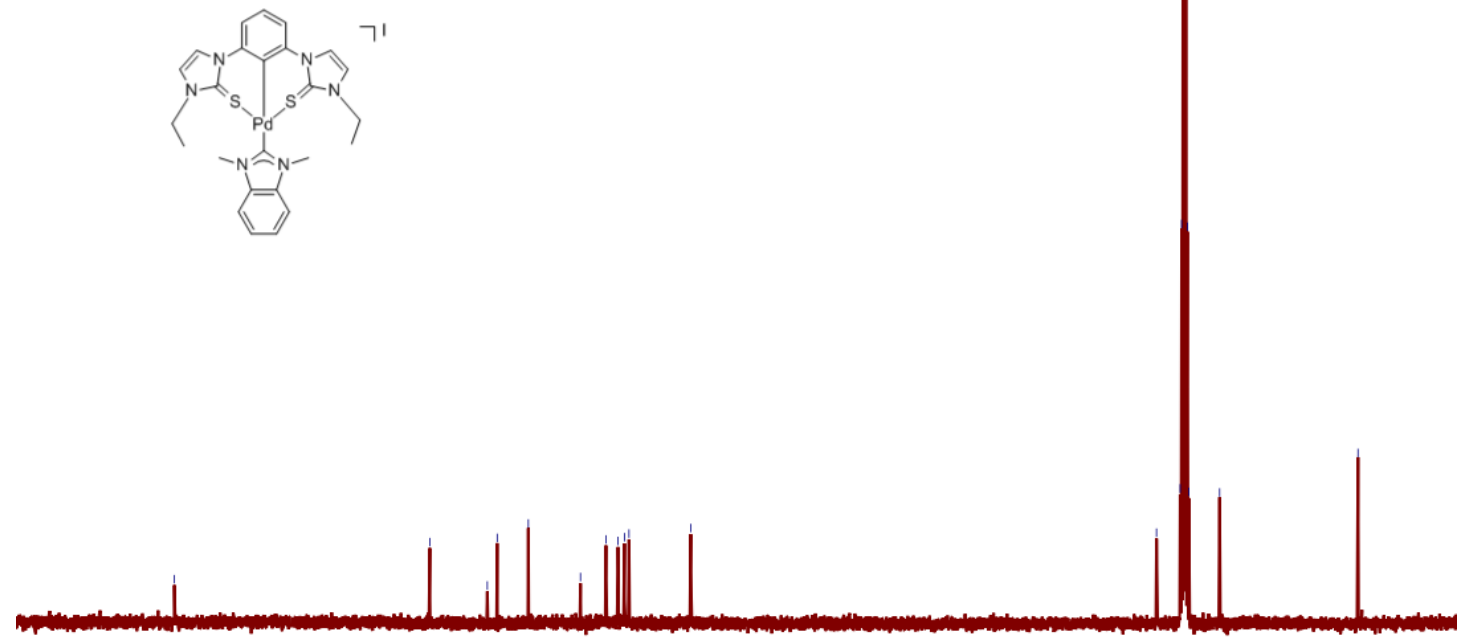

$\begin{array}{llllllllllllllllllll}200 & 190 & 180 & 170 & 160 & 150 & 140 & 130 & 120 & 110 & 100 & 90 & 80 & 70 & 60 & 50 & 40 & 30 & 20 & 10\end{array}$

Figure S8. The ${ }^{13} \mathrm{C}$ NMR of $2 \mathbf{b}\left(100 \mathrm{MHz}, \mathrm{DMSO}-\mathrm{d}_{6}\right)$ 


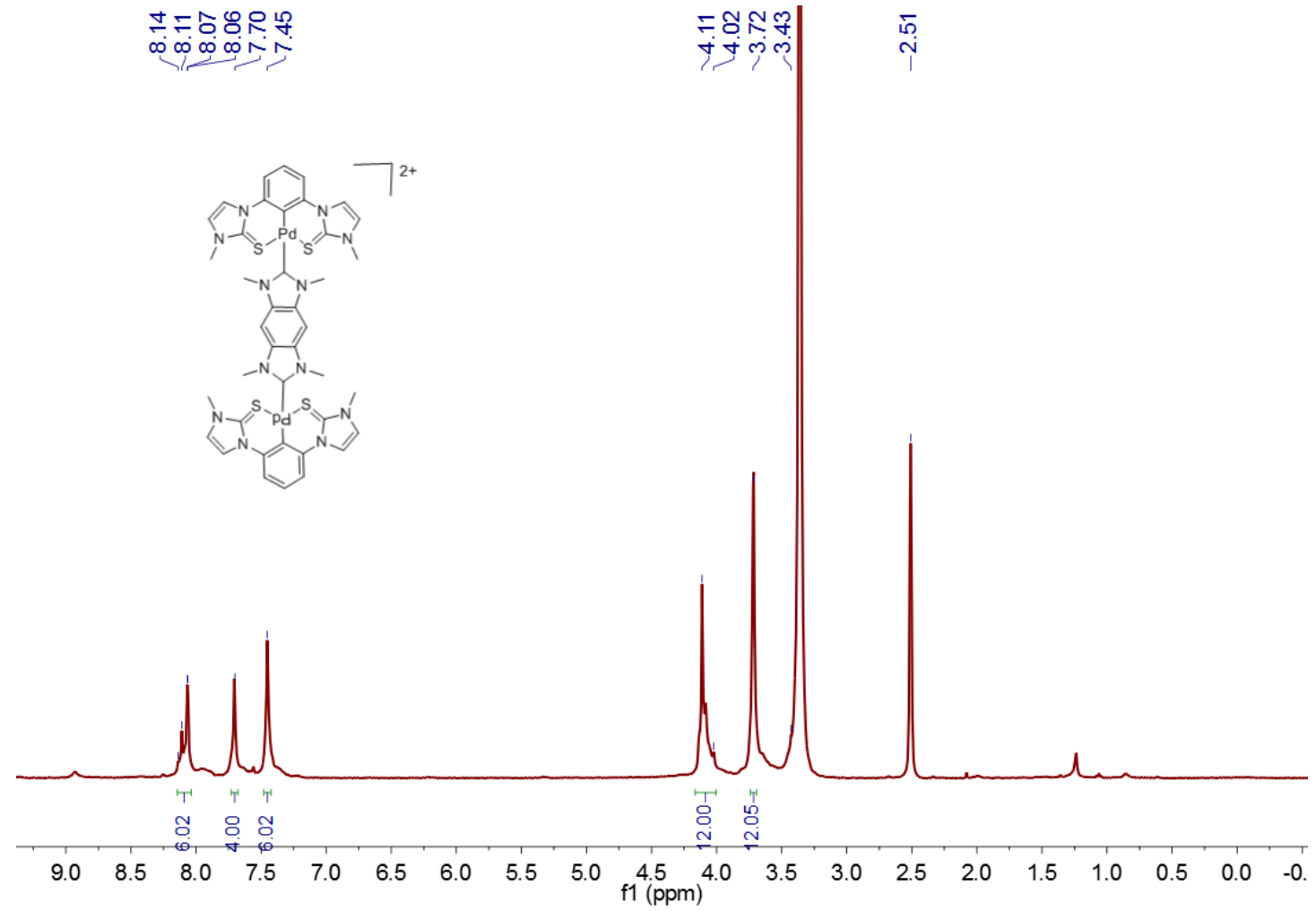

Figure S9. The ${ }^{1} \mathrm{H}$ NMR of 3a $\left(400 \mathrm{MHz}, \mathrm{DMSO}-\mathrm{d}_{6}\right)$

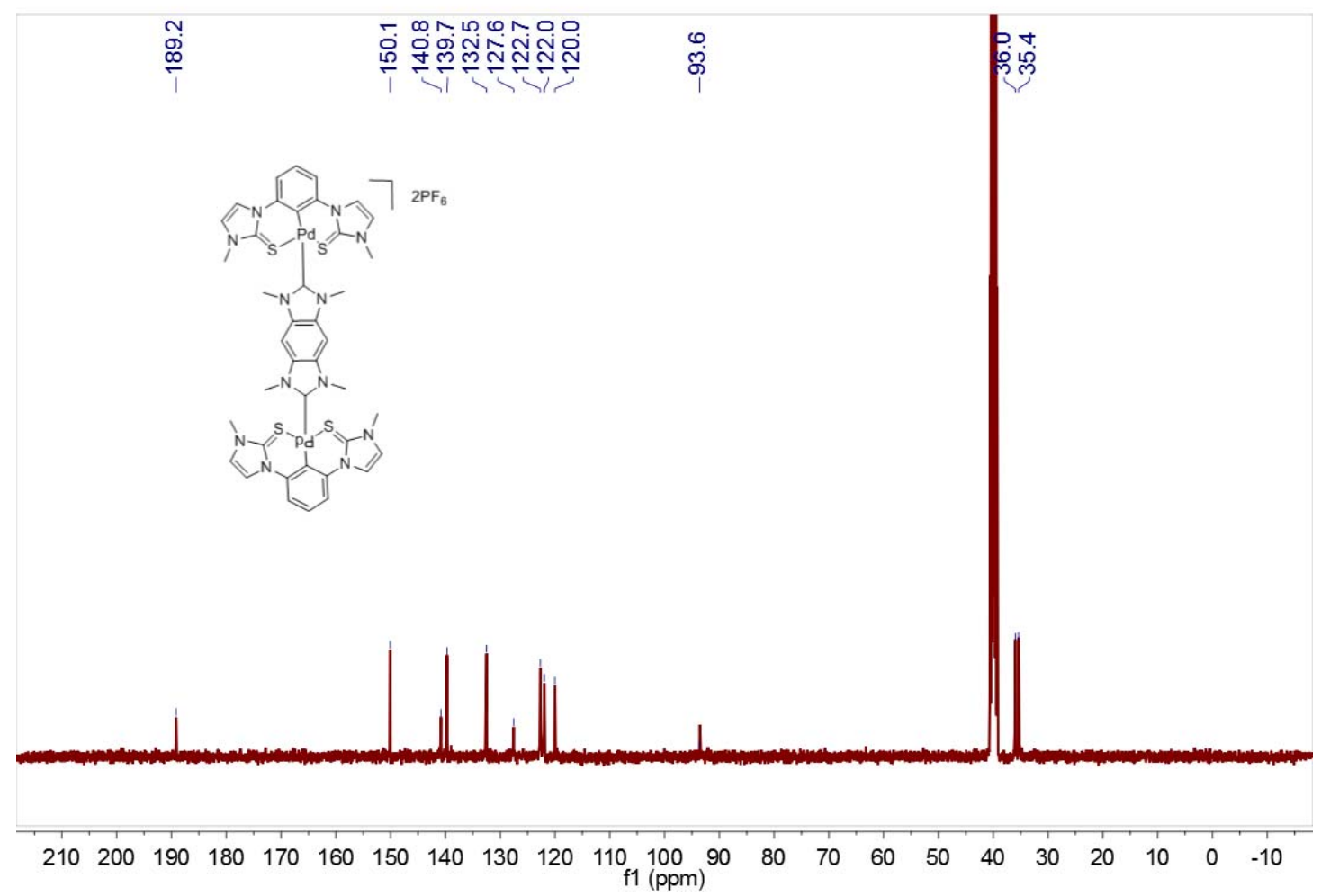

Figure S10. The ${ }^{13} \mathrm{C}$ NMR of 3a (100 MHz, DMSO-d 6 ) 


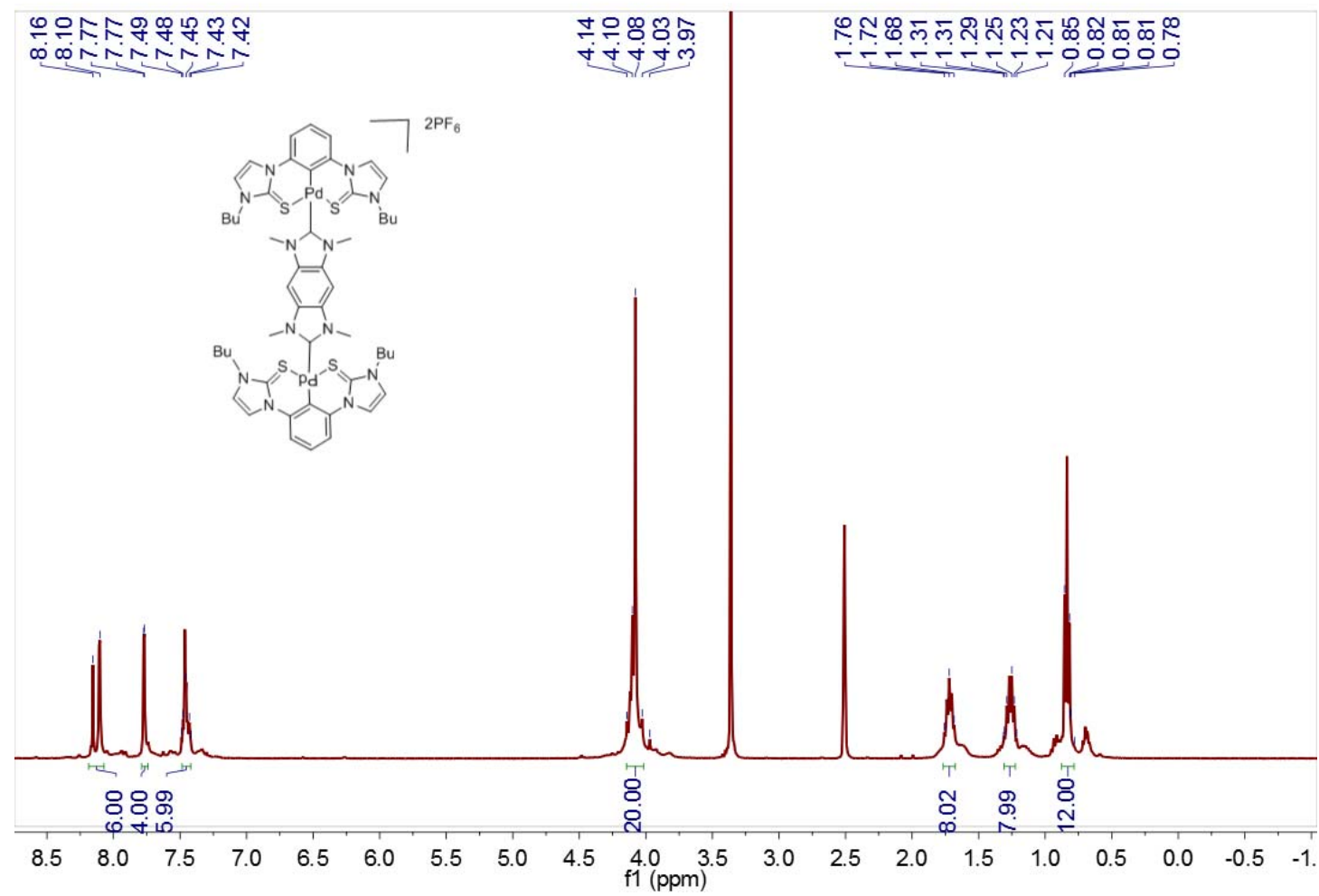

Figure S11. The ${ }^{1} \mathrm{H}$ NMR of $3 \mathbf{b}\left(400 \mathrm{MHz}, \mathrm{DMSO}-\mathrm{d}_{6}\right)$

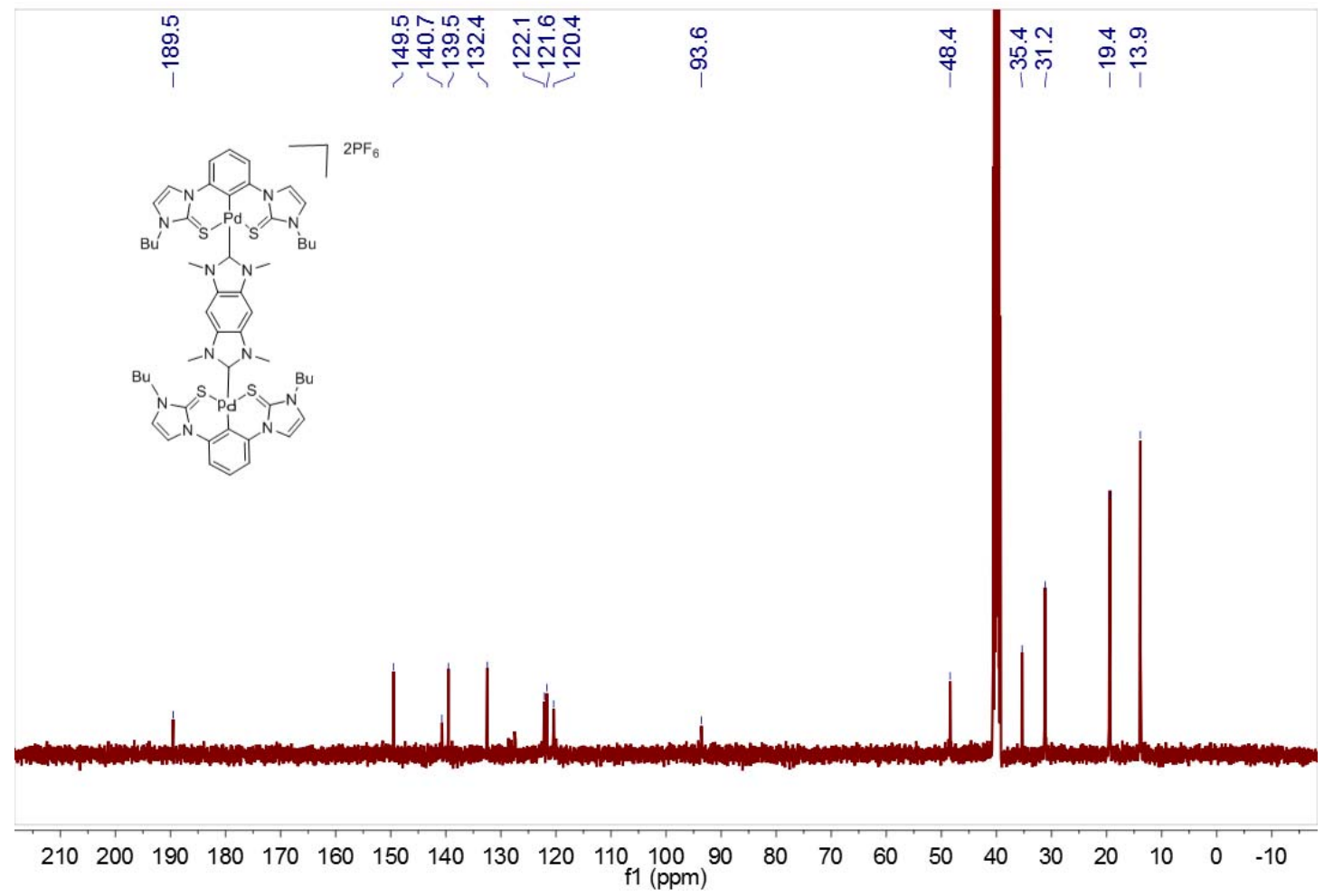

Figure S12. The ${ }^{13} \mathrm{C}$ NMR of $\mathbf{3 b}\left(100 \mathrm{MHz}, \mathrm{DMSO}-\mathrm{d}_{6}\right)$ 


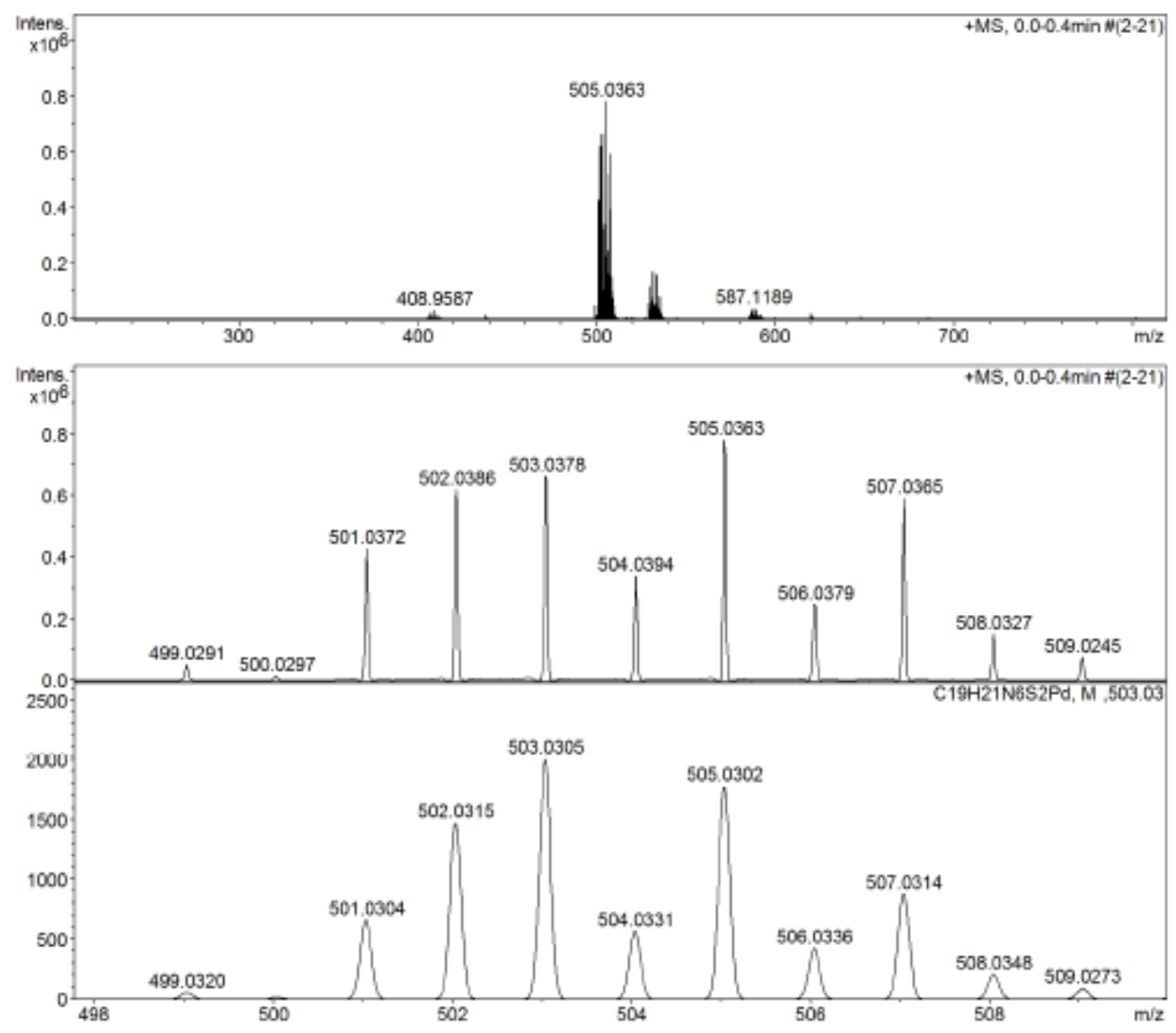

Figure S13 The ESI-MS spectra of complex Ia
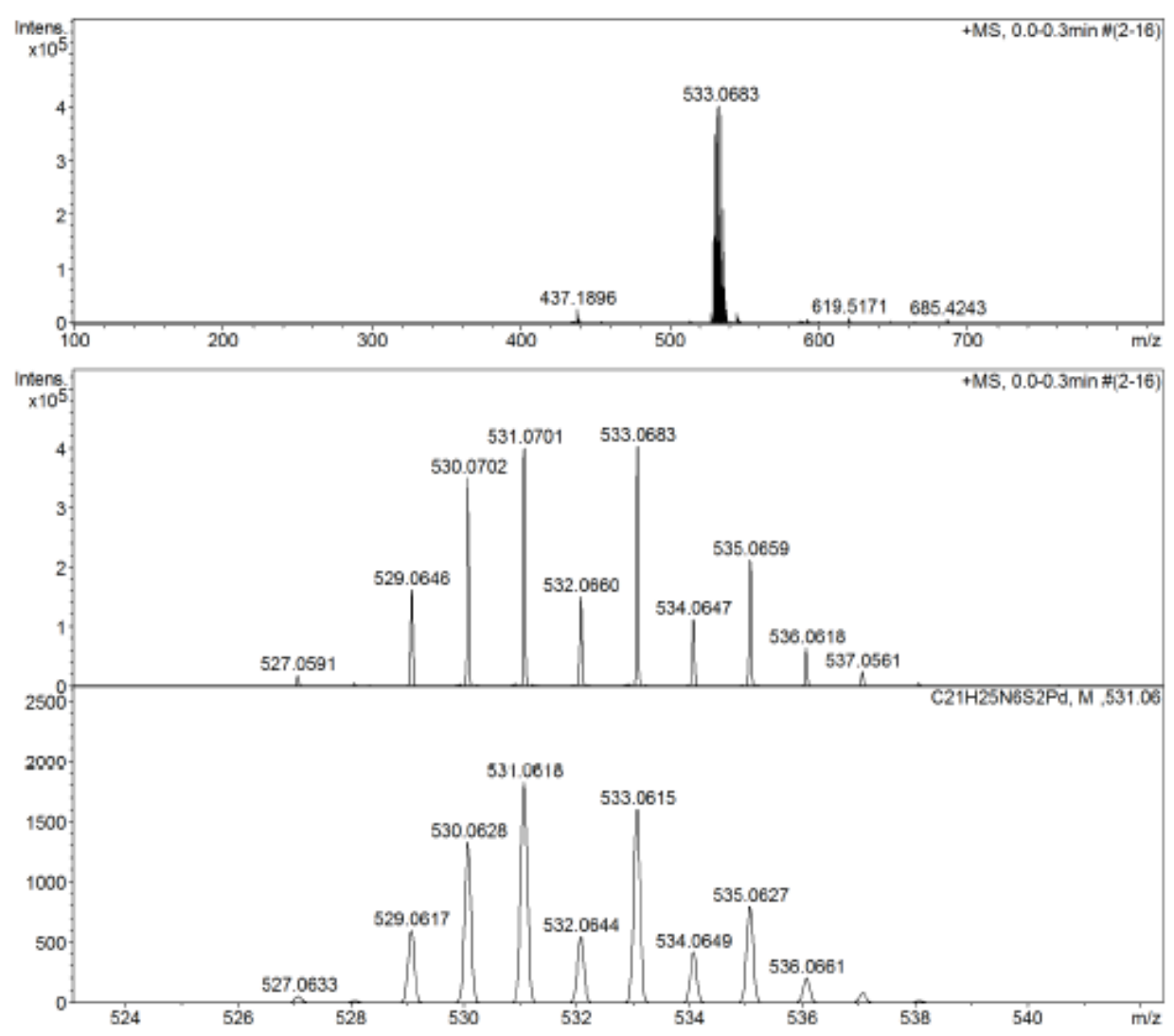

Figure S14. The ESI-MS spectra of complex lb

S10 

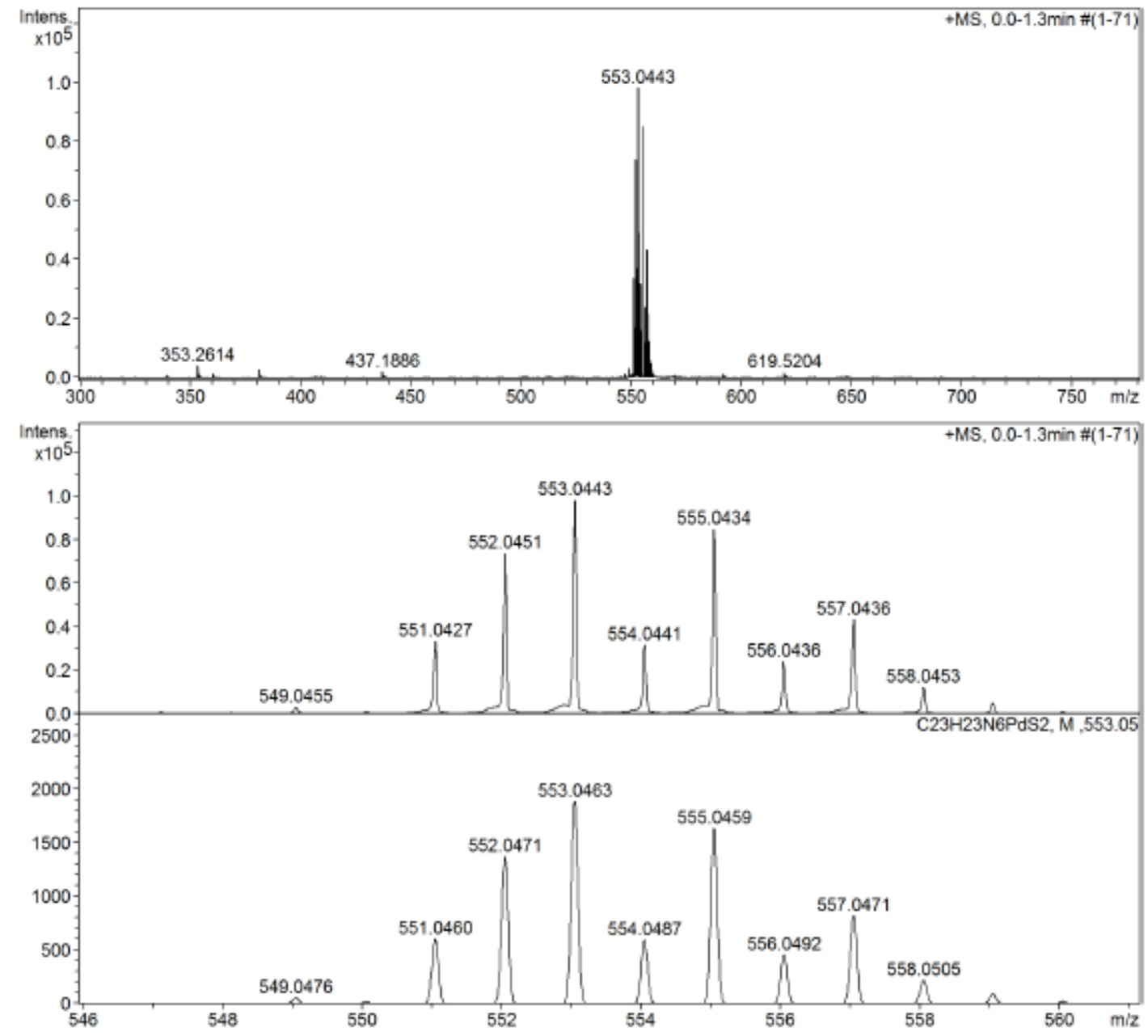

Figure S15. The ESI-MS spectra of complex 2a 


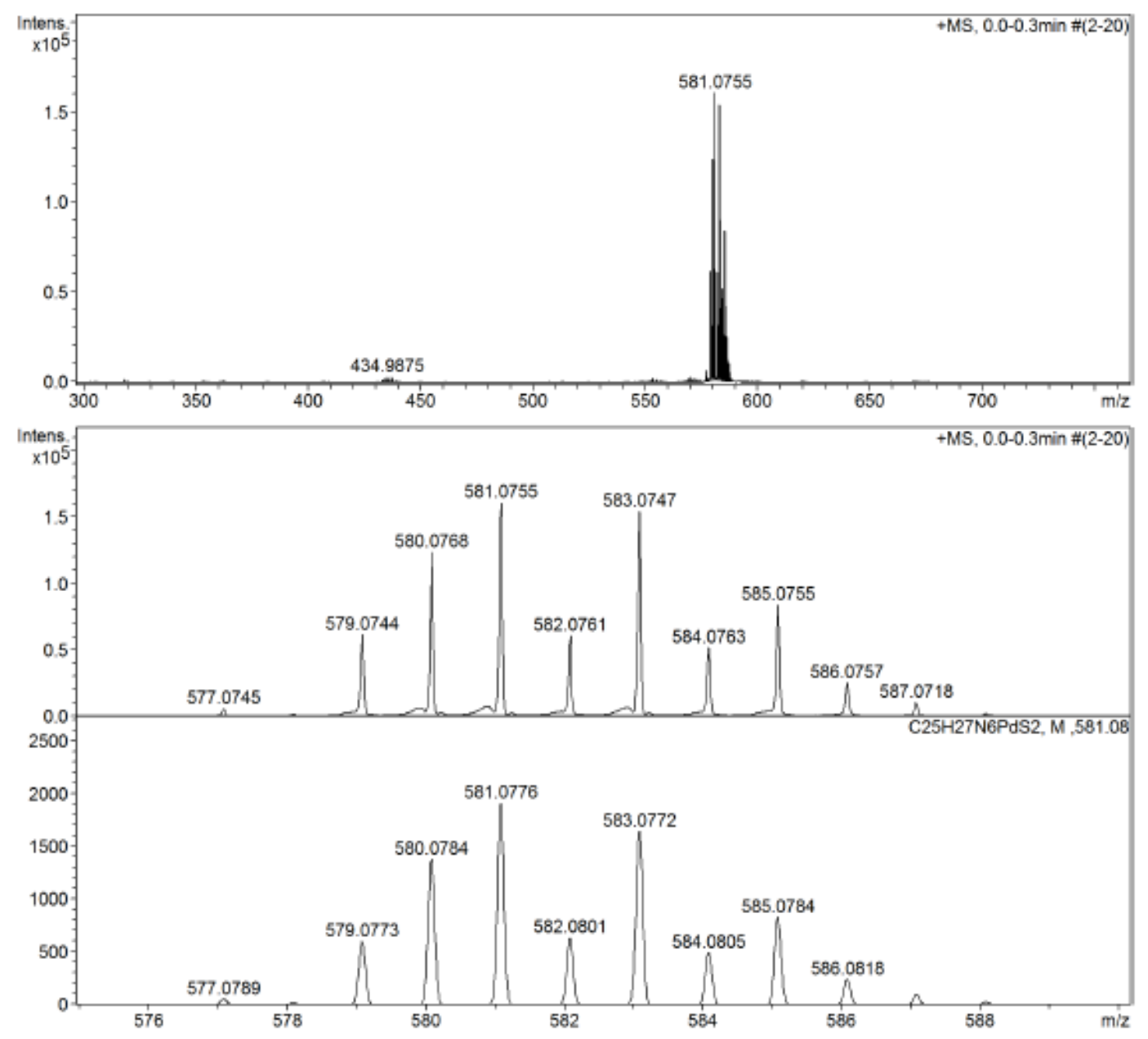

Figure S16. The ESI-MS spectra of complex 2b 


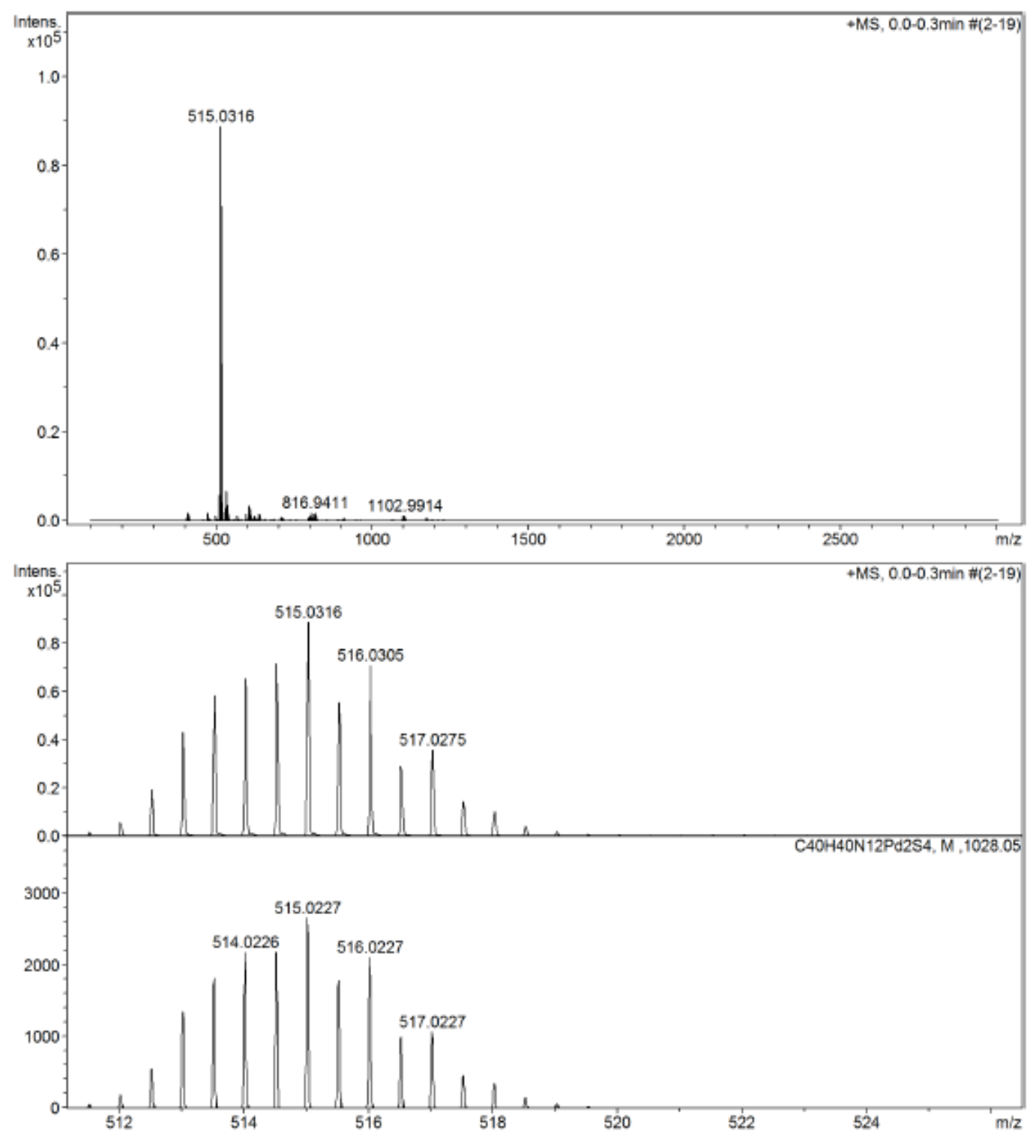

Figure S17. The ESI-MS spectra of complex 3a 

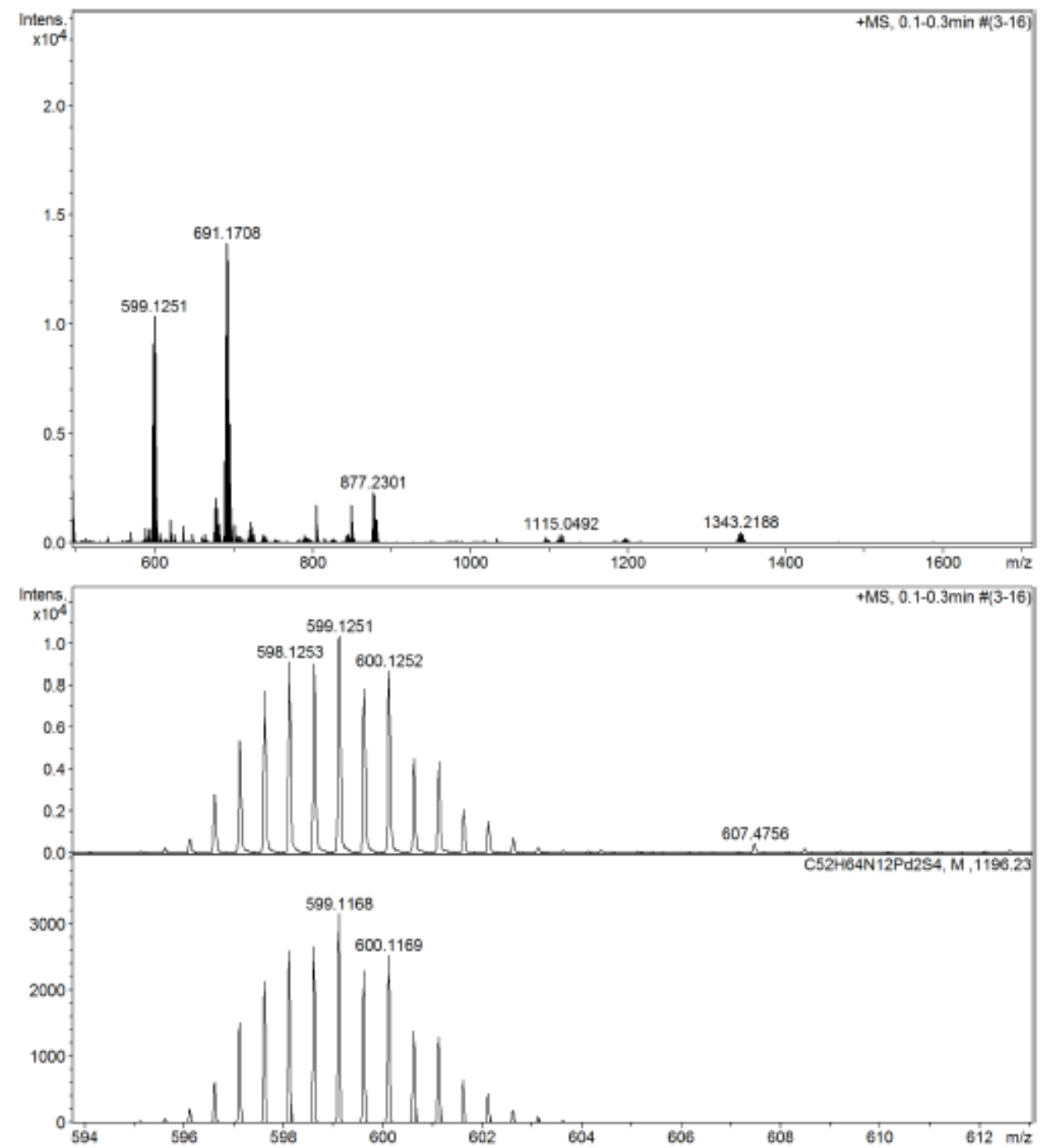

Figure S18. The ESI-MS spectra of complex 3b 